\title{
Does the real-time thermal damage estimate allow for estimation of tumor control after MRI-guided laser-induced thermal therapy? Initial experience with recurrent intracranial ependymomas
}

\author{
${ }^{*}$ Nitesh V. Patel, MD, ${ }^{1}$ Pinakin R. Jethwa, MD, ${ }^{2}$ Anil Shetty, PhD, ${ }^{3}$ and Shabbar F. Danish, MD1 \\ 1'Division of Neurosurgery, Rutgers-Robert Wood Johnson Medical School, New Brunswick, New Jersey; ${ }^{2}$ Department of \\ Neurological Surgery, Rutgers-New Jersey Medical School, Newark, New Jersey; and ${ }^{3}$ Visualase Inc., Houston, Texas
}

OBJECT Although control of intracranial ependymomas is highly correlated with degree of resection, it is unknown if the same is true for MRI-guided laser-induced thermal therapy (MRgLITT). The authors report their experience with MRgLITT for ependymoma and examine the utility of the real-time thermal damage estimate (TDE), a recent software advance, with respect to completeness of ablation and impact on tumor control. To the authors' knowledge, this is the largest single-center experience utilizing MRgLITT for recurrent ependymomas.

METHODS Five tumors in 4 patients were treated with the Visualase Thermal Therapy System. Two tumors were treated similarly on recurrence. Ablation was performed using a $980-\mathrm{nm}$ diode laser with a real-time image acquisition system. Single-plane TDEs were calculated and compared with the original lesion area to compute percentage area ablated (PAA). Volumetric analysis was performed, and percentage volume ablated (PVA) was estimated and correlated with the TDE. Tumor control was correlated with the TDE and volumetric data during treatment.

RESULTS Nine ablations were performed on 5 tumors, 2 of which had multiple recurrences. The average pretreatment lesion volume was $8.4 \pm 6.3 \mathrm{~cm}^{3}$, and the average largest 2D area was $5.3 \pm 2.7 \mathrm{~cm}^{2}$. The averaged TDE was $3.9 \pm 2.1$ $\mathrm{cm}^{2}$, average PAA was $80.1 \% \pm 34.3 \%$, and average PVA was $64.4 \% \pm 23.5 \%$. For subtotal ablations, average recurrence time was $4.4 \pm 5.3$ months; 1 adult case remains recurrence-free at 40 months. Using TDEs, the correlation between recurrence time and PAA was $r=0.93(p=0.01)$, and for PVA was $r=0.88(p=0.02)$. Furthermore, PVA and PAA were strongly correlated $(r=0.88, p=0.02)$.

CONCLUSIONS Through using the PAA, the real-time TDE correlated with the volume of ablation in this initial investigation. Furthermore, the TDE and volumetric data corresponded to the level of tumor control, with time to recurrence dependent on ablation completeness. MRgLITT may have a role in the management of recurrent ependymomas, especially with recent software advances.

http://thejns.org/doi/abs/10.3171/2014.10.PEDS13698

KEY WORDS ependymoma; laser therapy; magnetic resonance thermometry; stereotactic tumor ablation; oncology

$\mathrm{T}$ HE standard therapeutic approach for ependymomas has been surgery followed by radiotherapy, depend-

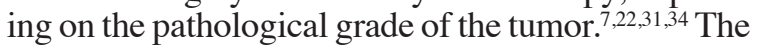
extent of resection greatly affects the prognosis for these patients. . $2,31,34$ Fractionated radiation and radiosurgery have been used for recurrent disease with variable long-term results. ${ }^{14}$ Chemotherapy has been used, but its role in the treatment paradigm of ependymomas has yet to be determined, as it has not shown to improve survival in comparison with radiation therapy alone. ${ }^{4,8,35}$ Despite advances in surgical techniques and adjuvant therapies, the 5-year survival for this disease remains approximately $35 \%-60 \% .{ }^{4}$

Magnetic resonance imaging-guided laser-induced thermal therapy (MRgLITT) is a minimally invasive

ABBREVIATIONS MRgLITT = MRI-guided laser-induced thermal therapy; PAA = percentage area ablated; PVA = percentage volume ablated; TDE = thermal damage estimate.

ACCOMPANYING EDITORIAL See pp 361-362. DOI: 10.3171/2014.8.PEDS14297.

SUBMITTED December 30, 2013. ACCEPTED October 23, 2014.

INCLUDE WHEN CITING Published online January 16, 2015; DOI: 10.3171/2014.10.PEDS13698.

DISCLOSURE Anil Shetty, PhD, is under the employ of and holds equity in Visulase Inc. No funding was obtained for this project from Visualase Inc., and Dr. Shetty was strictly involved in writing only the Methods portion of the manuscript, specifically the portion regarding the mathematics behind MRgLITT.

* Drs. Patel and Jethwa contributed equally to this work. 
method of treating neoplasms that has been used with success in intracranial tumors, and several types of extracranial tumors, including liver metastases, squamous cell carcinomas of the head and neck, invasive breast cancers, adrenal metastases, lung metastases, and prostate cancers. ${ }^{6,12,18,26,29,30,36}$ Energy in the form of light is emitted through a saline- or water-cooled catheter and converted to thermal energy within the target tissue. ${ }^{10,37}$ The resulting thermal coagulation and damage to intracellular proteins and DNA ultimately leads to cell death..$^{10,37}$ This system can potentially be used to treat tumors that may otherwise not be amenable to other conventional therapies. The addition of MRI to LITT allows the use of real-time MRI for planning, guidance, and thermal monitoring during the ablation. $1.6,9,13,17$

Despite the advent of MRgLITT over the last 25 years, this approach has failed to gain wide acceptance in the therapy of malignant and recurrent brain tumors., ${ }^{2,5,28} \mathrm{Re}-$ cent advances in the technique have generated a renewed interest in applying MRgLITT to intracranial disease. The improvement in imaging resolution, time to image acquisition, ablation transition zones, and the ability to estimate the thermal damage in real time are all factors that have enhanced the safety and efficacy of MRgLITT for neurosurgical pathologies.

The real-time delivery of thermal therapy when using the Visualase Thermal Therapy System (Visualase Inc.) is quantified based on the thermal damage estimate (TDE; Fig. 1), which is calculated based on the Arrhenius rate process model. Time and temperature profiles at each pixel are used to calculate an estimated permanent damage zone based on this model, described in the equation,

$\Omega(\mathrm{t})=\int_{0}^{t} A e^{\frac{-E_{a}}{R T(t)}} d t$

in which $\mathrm{E}_{\mathrm{a}}$ and $\mathrm{A}$ are the activation energy and frequency factor (constant representing the frequency of collisions when the molecules are properly oriented), respectively; in general, these are tissue-dependent constants. $T(t)$ is the tissue temperature over time, $\mathrm{R}$ is the universal gas constant, and t is time. In the Visualase software, voxels are assumed to have reached the threshold for thermal damage when $\Omega \geq 1$ and the voxels are displayed in orange. The area of damage can then be calculated from the software representation of irreversible thermal ablation.

In this study, we investigate our early experience in 4 patients with recurrent anaplastic ependymomas. We provide clinical scenarios in which we believe that MRgLITT was an appropriate therapy. We hypothesized that the real-time 2D TDE, a new software advent, would correlate with the volume of ablation, and give insight into the ability to achieve long-term control using this technique in these difficult cases.

\section{Methods}

\section{Surgical Technique}

Patients for this study were retrospectively analyzed

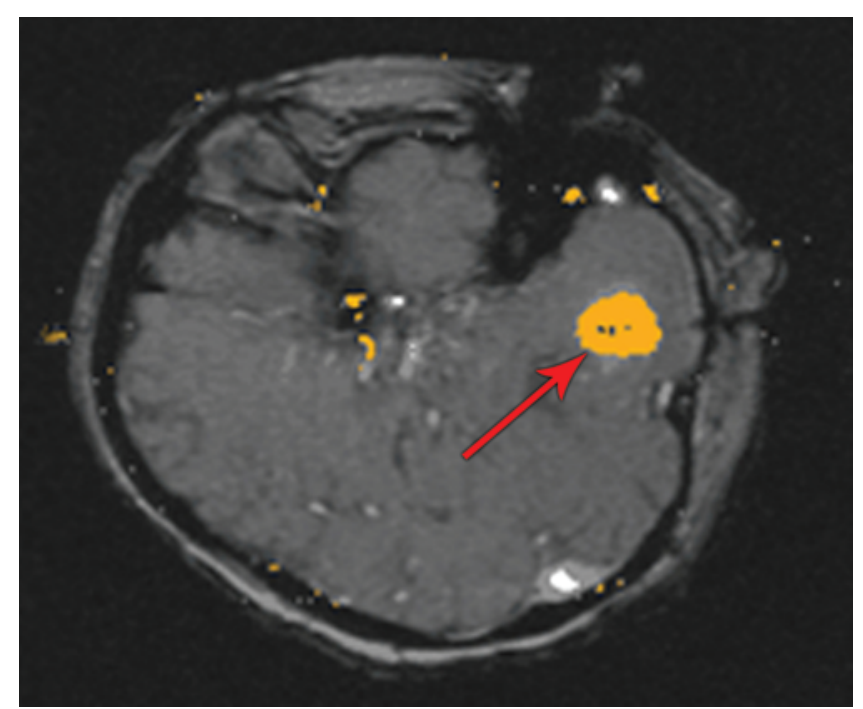

FIG. 1. Axial MR image showing the thermal damage estimate (TDE) for an example case with a left-sided posterior fossa mass. The area ablated is shaded in orange (arrow) and overlays the actual lesion's area. Additional small areas of orange noted are due to artifact on the image. Figure is available in color online only.

based on the diagnosis of progressive recurrent ependymoma. A multidisciplinary neurooncology board determined appropriateness of the procedure, and all patients were prospectively enrolled in an Institutional Review Board-approved study with informed consent. The Visualase Thermal Therapy System was used in all cases.

The details of the laser thermal ablation technique used by our institution have been previously reported.12 Briefly, stereotactic MRI is performed on the morning of surgery for planning, and the patient is then taken to the operating room. Using navigation software (Stealth S7, Medtronic, Inc.), registration and trajectory planning are performed. After confirmation of position and trajectory, a $3.2-\mathrm{mm}$ power drill bit is used to create a bur hole. The plastic bone anchor (Visualase, Inc.) is placed into the skull through the fixed trajectory, and once in place, the laser applicator is passed. When the appropriate depth is reached, the applicator is secured to the bone anchor. The patient is transferred to the MRI unit for the ablation.

A 3D, T1-weighted, fast spoiled gradient image is obtained and the Visualase system is connected. A working projection is then determined and a reference image is obtained. During the procedure the Visualase system provides real-time temperature feedback. Temperature limits are set at the borders of the lesion or near critical structures to avoid thermal damage to normal brain.

Thermal energy is delivered and the system provides information on the tissue that is irreversibly damaged on the screen. Operative parameters such as power, time of ablation, and position of the tip of the laser catheter were adjusted at the discretion of the surgeon (S.F.D.) to safely ablate the lesion. The TDE is superimposed on the preablation lesion area to visually estimate the extent of ablation. The procedure was determined to be complete when the surgeon believed that maximal ablation had been reached. All patients were followed-up with serial contrast-enhanced MRI. The presence of recurrence was 
assessed radiologically and by determination of a multidisciplinary neurooncology board.

\section{Statistical and Mathematical Measures}

All pretreatment tumor areas were calculated based on the slice with the widest diameters in the plane of treatment. OsiriX DICOM Viewer's (version 3.9.4; Pixmeo SARL) region-of-interest tracing tool was used for preablation areas. The TDE was calculated and represented the $2 \mathrm{D}$ estimated area of ablation. The number of damage voxels was converted into damage area $\left(\mathrm{mm}^{2}\right)$ by using the formula,

$$
\mathrm{TDE}=(\text { no. of pixels }) \times(\mathrm{FOV} \times \mathrm{FOV}) /(256 \times 256)
$$

in which FOV refers to the field of view. All pre- and postablation lesion volumes were measured using polygonal tracing with fusion via OsiriX DICOM Viewer, and all calculations were primarily made using T1-weighted MRI with Gd enhancement. As we have previously reported, the ablated region was identified as that within the boundaries of the enhanced periphery. ${ }^{24}$ Percentage area ablated (PAA) was calculated as a ratio of TDE area to pre-ablation tumor area, and percentage volume ablated (PVA) was calculated as a ratio of volume ablated to preablation tumor volume. This allowed normalization of the TDE and volume ablated to the pre-ablation tumor measurements. Pearson correlation was used to determine the relationship between PAA, PVA, and time to recurrence.

\section{Results}

A total of 9 MRgLITT procedures were performed to treat 5 tumors in 4 patients. Two female adults and 2 male children were included. The average age of the 4 patients was $32 \pm 22$ years old. Each patient's clinical history, laser procedure, and clinical course are described below.

\section{Case 1}

This 59-year-old woman had undergone 2 craniotomies and fractionated radiotherapy for a recurrent subcallosal anaplastic ependymoma. The patient was being followed and had evidence of a small nodule of recurrent tumor in the surgical bed. She was initially observed, but when the nodule showed evidence of progression, we advocated for treatment. The options of repeat craniotomy, stereotactic radiosurgery, and MRgLITT were offered. The patient was adamantly against another open surgery and opted for MRgLITT after a discussion of available options.

The pre-ablation tumor is shown in Fig. 2A and measured $0.6 \mathrm{~cm}^{3}$. Her operative parameters, including TDE, are shown in Table 1. Her immediate postablation image is shown in Fig. 2B. The PAA and PVA were $155 \%$ and $97 \%$, respectively. The patient tolerated the procedure well and was discharged the next day. She has been followedup using serial imaging for 40 months, and has had no radiological signs of recurrence. Her follow-up imaging is shown in Fig. 3A-C. There has been a significant decrease in postablation enhancement over the course of her follow-up, as would be expected. She continues to work as a teacher and has no significant deficits affecting her daily function.

\section{Case 2}

This 42-year-old woman had undergone an endoscopic third ventriculostomy with biopsy, followed by a supracerebellar infratentorial approach for a posterior third-ventricular anaplastic ependymoma. She underwent subtotal resection followed by observation. She presented with radiological progression, and decided to undergo MRgLITT after a discussion of available options, which included repeat resection and MRgLITT.

The pre-ablation tumor is shown in Fig. $2 \mathrm{C}$ and measured $3.2 \mathrm{~cm}^{3}$. The PAA and PVA were both $88 \%$. Her postablation lesion is shown in Fig. 2D. The patient was discharged the next day without neurological deficits. The tumor showed radiological evidence of recurrence at 14 months, yet she remained neurologically intact. Her follow-up images are shown in Fig. 3D-F. After counseling the patient about the prognosis without further treatment, she underwent a transcallosal approach to the tumor, in which a subtotal resection was achieved. She underwent fractionated radiation after surgery and continues to be followed. The patient remains independent in her daily activities.

\section{Case 3}

This 13-year-old boy had a history of multiple craniotomies, chemotherapy trials, radiation therapy, and multiple endoscopic resections. He initially presented with a fourth-ventriclular anaplastic ependymoma that then metastasized to the third and right lateral ventricles. He presented to our institution with progression of a right frontal ventricular and third ventricular mass. He was not being offered any intervention at any other institution for these progressive tumors. MRgLITT was offered to the patient to attempt palliative control. The tumors were initially ablated in separate sessions. On recurrence, both tumors were treated simultaneously. The right frontal ventricular tumor (Case 3A, Table 1) measured $11.8 \mathrm{~cm}^{3}$ (Fig. 2E). The PAA and PVA were $67 \%$ and $62 \%$, respectively. The ablation was uneventful and the patient was discharged the next day. One month later, the third ventricular tumor underwent ablation (Case 3B). At this point, the right frontal tumor remained stable. The third ventricular tumor measured $3.5 \mathrm{~cm}^{3}$ (Fig. 2G). The PAA and PVA were $52 \%$ and $60 \%$, respectively. The patient was discharged the following day after this ablation. Postablation images for each of these procedures are shown in Fig. $2 \mathrm{~F}$ and $2 \mathrm{H}$.

Radiological recurrence of both tumors was noted at 2 months. Both tumors were treated with MRgLITT at recurrence simultaneously, with each tumor requiring a separate laser catheter (Case 3C). The PAA for the right frontal and third ventricular tumors was $60 \%$ and $51 \%$, respectively. The PVA for the right frontal and third ventricular tumors was $45 \%$ and $40 \%$, respectively. The patient tolerated the procedure well and was discharged the following day. The reason for the subtotal ablation was to account for the acute postablation edema, which resolved several weeks later. Larger ablations could have lead to pressure symptoms that could have compromised cognitive and functional aspects. Therefore, these decisions were made to balance treatment goals and safety.

Recurrence was noted again at 2 months. The patient 

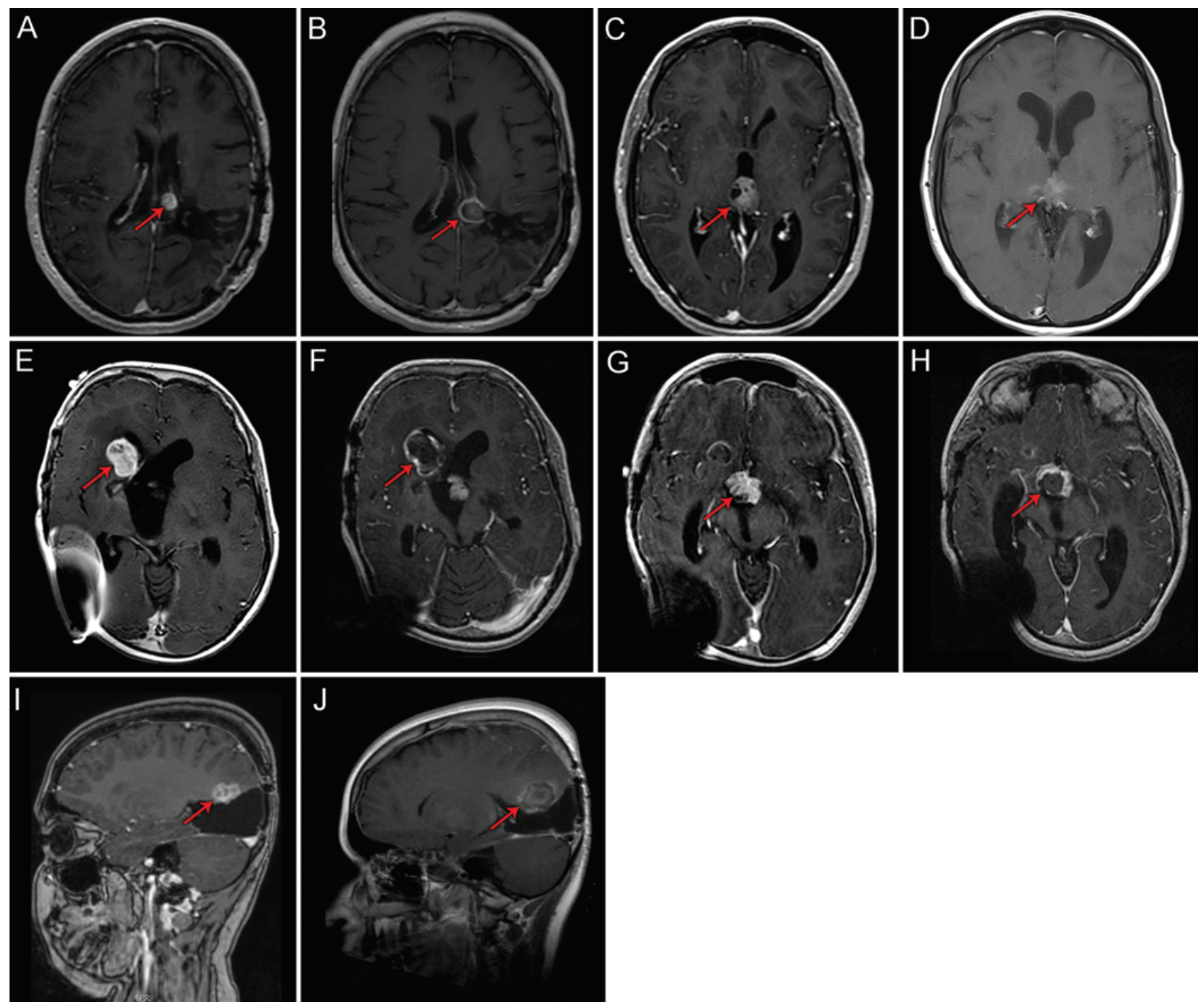

FIG. 2. Axial (A-H) and sagittal (I and J) Gd-enhanced T1-weighted MR images for Case 1 (A and B), Case 2 (C and D), Case 3 ( $\mathbf{E}$ and $\mathbf{F}$, right frontal lesion; $\mathbf{G}$ and $\mathbf{H}$, third ventricle lesion), and Case 4 (I and $\mathrm{J}$ ). In each pair of images, the left panel shows pre-ablation lesions (arrows), and the right panel shows postablation images (arrows). Figure is available in color online only.

TABLE 1. MRgLITT operative parameters and lesion measures

\begin{tabular}{|c|c|c|c|c|c|c|c|c|c|}
\hline \multirow{3}{*}{$\begin{array}{c}\text { LITT Parameter } \\
\text { Lesion location }\end{array}$} & \multicolumn{9}{|c|}{ Patient } \\
\hline & \multirow{2}{*}{$\frac{1}{\text { Splenium }}$} & \multirow{2}{*}{$\begin{array}{c}2 \\
3 \mathrm{~V}\end{array}$} & \multirow{2}{*}{$\frac{3 \mathrm{~A}}{\mathrm{RFLV}}$} & \multirow{2}{*}{$\frac{3 \mathrm{~B}}{3 \mathrm{~V}}$} & \multicolumn{2}{|c|}{$3 \mathrm{C}$} & \multicolumn{2}{|c|}{$3 \mathrm{D}$} & \multirow{2}{*}{$\begin{array}{c}4 \\
\text { POC }\end{array}$} \\
\hline & & & & & RFLV & $3 \mathrm{~V}$ & RFLV & $3 \mathrm{~V}$ & \\
\hline Pre-LITT area $\left(\mathrm{cm}^{2}\right)$ & 1.2 & 4.5 & 5.9 & 4.2 & 7.9 & 3.9 & 8.5 & 9.1 & 2.9 \\
\hline TDE $\left(\mathrm{cm}^{2}\right)$ & 1.9 & 3.9 & 3.9 & 2.2 & 4.8 & 2 & 5.4 & 7.9 & 2.9 \\
\hline PAA (\%) & 155 & 88 & 67 & 52 & 60 & 51 & 63 & 87 & 103 \\
\hline Pre-LITT volume $\left(\mathrm{cm}^{3}\right)$ & 0.6 & 3.2 & 11.8 & 3.5 & 17.9 & 8.6 & 17.1 & 9.8 & 3.1 \\
\hline Ablated volume $\left(\mathrm{cm}^{3}\right)$ & 0.6 & 2.8 & 7.3 & 2.1 & 8.1 & 3.4 & 7.2 & 4.8 & 3 \\
\hline PVA (\%) & 97 & 88 & 62 & 60 & 45 & 40 & 42 & 49 & 97 \\
\hline Recurrence (mos) & NR & 14 & 2 & 2 & 2 & 2 & NR & NR & NR \\
\hline
\end{tabular}

$3 V=$ third ventricle; $N R=$ no noted recurrence $\mathrm{POC}=$ posterior occipital; RFLV = right frontal lateral ventricle. 

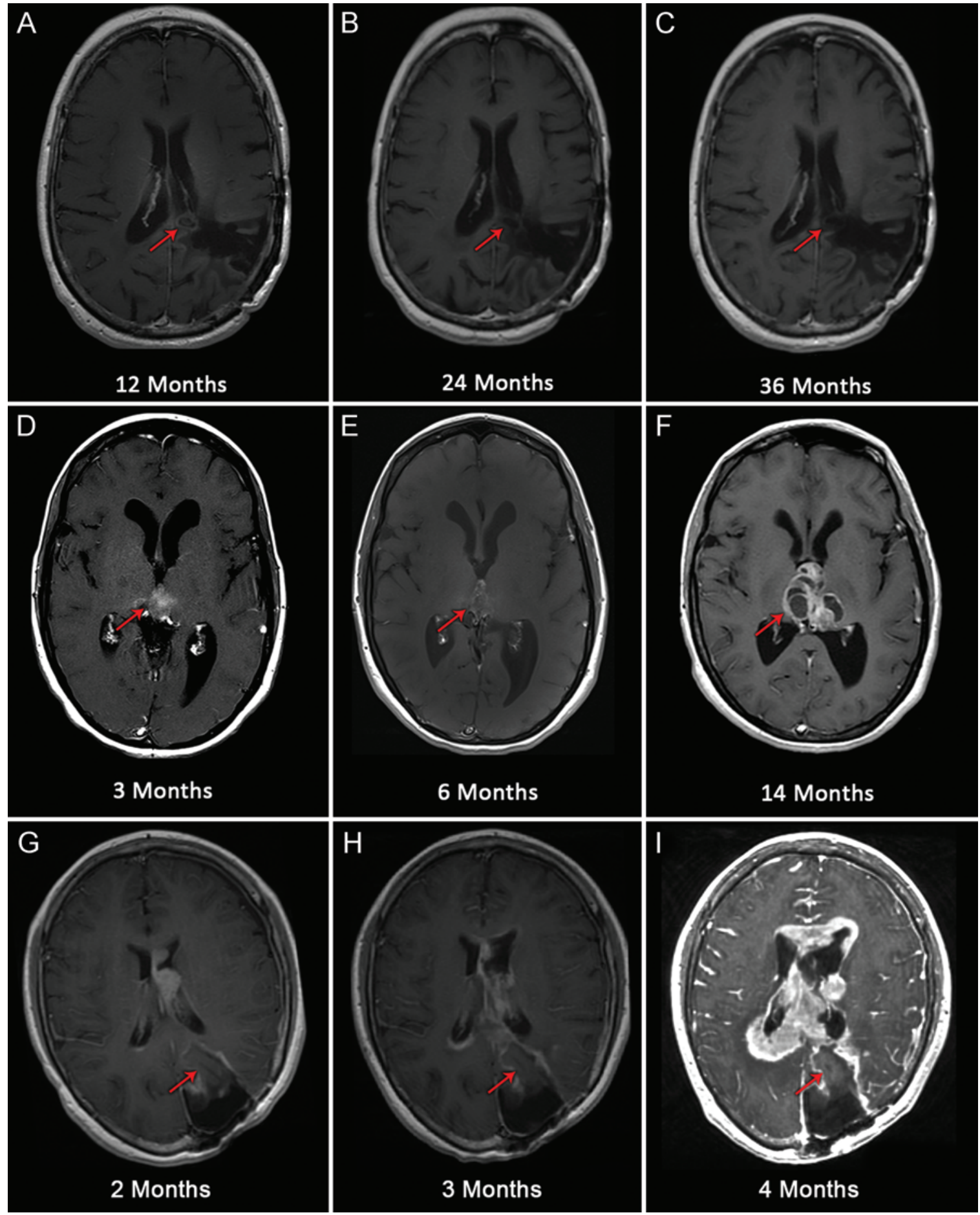

FIG. 3. Axial Gd-enhanced T1-weighted follow-up MR images for Cases 1 (A-C), 2 (D-F), and 4 (G-I). The arrow in each image indicates the region of interest. Figure is available in color online only.

remained at his neurological baseline, and the parents consented to a third procedure. Both tumors were treated simultaneously (Case 3D), but the third ventricular tumor required 2 lasers due to its size and geometry. The PAA for the right frontal and third ventricular tumors was $63 \%$ and $87 \%$, respectively. The PVA for the right frontal and third ventricular tumors was $42 \%$ and $49 \%$, respectively. After the procedure, the patient developed diabetes insipi- dus and acute metabolic abnormalities, including hyponatremia and hypokalemia. Despite stabilizing the diabetes insipidus and correcting the metabolic abnormalities, the patient never regained his preoperative neurological status. He was minimally conversant, and although able to walk, was unable to perform other basic daily activities. The patient's family elected to initiate hospice care, and the patient died several weeks later. 


\section{Case 4}

This 15 -year-old boy had a history of 5 craniotomies for recurrent anaplastic ependymoma within the left parietal lobe. He had exhausted radiation and chemotherapeutic options, and presented with radiological progression of disease at the resection margin. He also had additional smaller lesions in the left parietal lobe. The patient was referred to our institution for evaluation, and after we reviewed his images, the options of repeat craniotomy and MRgLITT were discussed. Having undergone many surgeries in the past, the patient and his parents were not amenable to another craniotomy. The patient and parents consented to MRgLITT after a full discussion of available options. The tumor measured $3.11 \mathrm{~cm}^{3}$ and was located in the superior portion of the previous left-sided parietooccipital resection bed (Fig. 2I). The PAA and PVA were 103\% and 97\%, respectively. His postablation image is shown in Fig. $2 \mathrm{~J}$. The patient was discharged the following day at his neurological baseline and was followed with serial MRI imaging. He showed evidence of metastasis at 4 months, although his ablated tumor remained stable (Fig. 3G-I). The patient was placed on hospice and subsequently died approximately 1 month later.

\section{Case Analyses}

For all patients, the average pre-ablation area was 5.3 $\pm 2.7 \mathrm{~cm}^{2}$ and average pre-ablation volume was $8.4 \pm 6.3$ $\mathrm{cm}^{3}$. The average trajectory length was $128.2 \pm 10.3 \mathrm{~mm}$. All lasers were determined to have optimal positioning (within the center of the intended ablation). The average power used for ablation was $10.5 \pm 0.8 \mathrm{~W}$. The mean total ablation time was $14.7 \pm 8.9$ minutes. The average TDE was $3.9 \pm 2.1 \mathrm{~cm}^{2}$ and average ablated volume was $4.4 \pm 2.7$ $\mathrm{cm}^{3}$. The average PAA was $80.1 \% \pm 34.3 \%$ while the average PVA was $64.4 \% \pm 23.5 \%$. The details of the ablations are summarized in Table 1. For the tumors that recurred, the average time to recurrence was $4.4 \pm 5.3$ months.

Analysis of the relationship between PAA, PVA, and time to recurrence was also performed. Only cases that recurred were included in the correlation analysis (Cases 2 and 3). For Case 3, each recurrence was treated as a separate tumor. There was a strong correlation between PAA and time to recurrence $(r=0.93, p=0.01)$. The relationship between PVA and time to recurrence was also significant $(\mathrm{r}=0.88, \mathrm{p}=0.02)$. A good correlation between PAA and PVA was also noted $(r=0.88, p=0.02)$. Based on these relationships, a curve was generated for each correlation with a line of best fit (Fig. 4).

Two of the 4 patients (both children) have died since the procedure. Their survival was 10 months (Case 3) and 5 months (Case 4). The 2 adult patients are still living: their follow-up and survival after the procedure has been 40 months (Case 1) and 35 months (Case 2).

\section{Discussion}

It is fairly well established that gross-total or near-total resections of intracranial ependymomas increase both progression-free and overall survival.,3,20,23,25,33 Because cytoreduction plays such a pivotal role, ependymoma is a "surgical disease" by nature. As such, subtotally resected

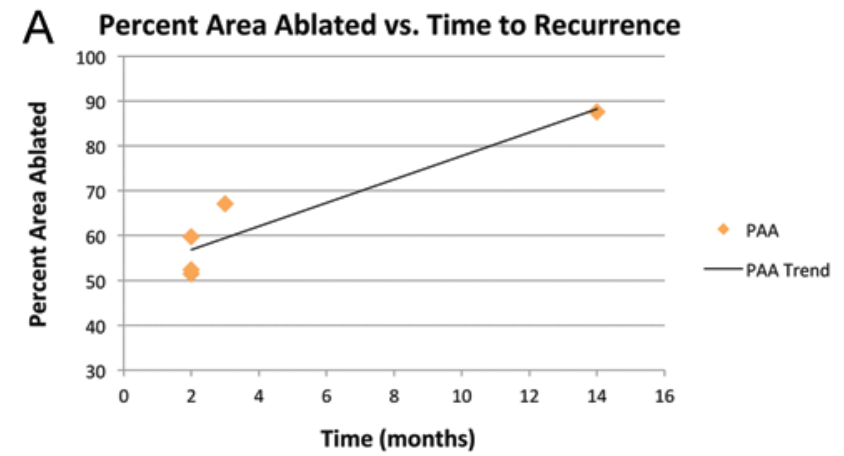

B Percent Volume Ablated vs. Time to Recurrence

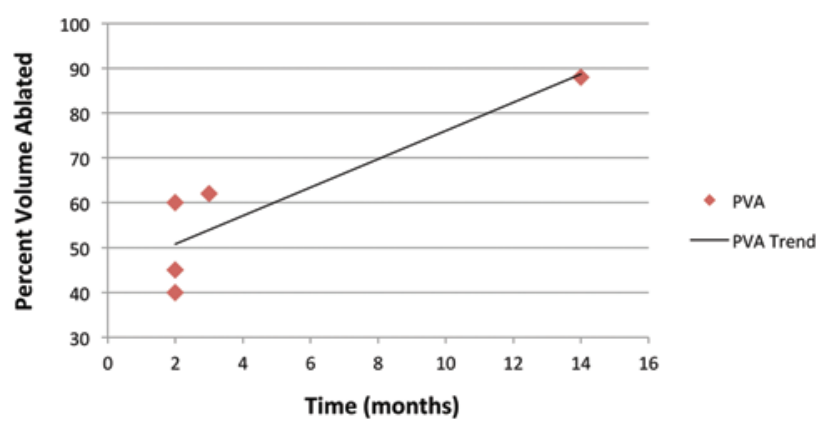

C Percent Volume Ablated vs. Percent Area Ablated

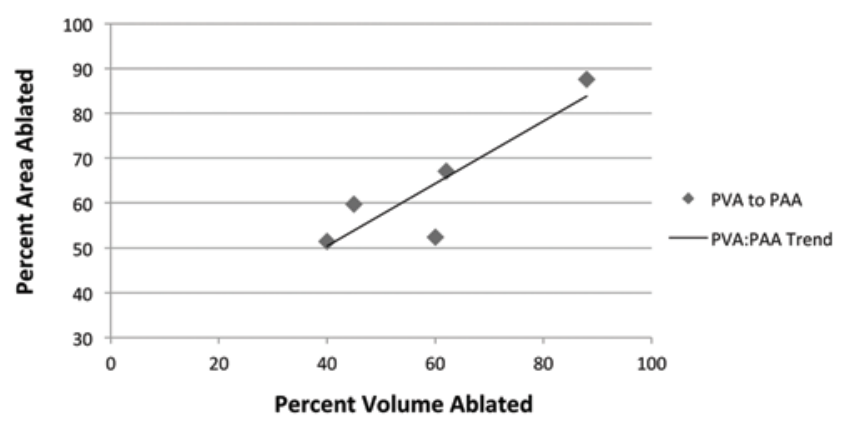

FIG. 4. Graphical comparisons of PAA and PVA with recurrence time. A: Comparison of PAA to recurrence time. B: Comparison of PVA to recurrence time. C: Graph showing the relationship between PAA and PVA. Both PAA and PVA correlated closely with recurrence time, and PAA and PVA correlated closely with each other. Figure is available in color online only.

and high-grade cases typically receive adjuvant treatments, such as radiation and chemotherapy. ${ }^{11,16,27}$ Stereotactic radiosurgery is a therapeutic option for intracranial ependymomas and has been shown to be effective in achieving palliative control in recurrent cases. ${ }^{15,19,32}$ Chemotherapy, although usually employed as a salvage option, has not been shown to provide consistent long-term control of these tumors. ${ }^{3}$ Thus, in cases for which resection, stereotactic radiosurgery, or chemotherapy fail, exploration of new options is necessary.

In this paper, we report our experience using the Visualase Thermal Therapy System to treat complex or difficultto-treat recurrent ependymoma. The 9 tumors treated in 4 patients represent the spectrum of problems often faced with recurrent ependymomas. In addition, we investigate whether the TDE is a reliable marker for control of these 
neoplasms. The TDE provides the operator with real-time estimations of irreversibly damaged tissue so that maximal tumor ablation with minimal damage to normal brain can be achieved during therapy. We hypothesized that MRgLITT can provide meaningful control in certain ependymomas, and that the TDE is correlated to that control.

\section{Patient Considerations}

Although asymptomatic on presentation, the patient in Case 1 had a small volume recurrence, and had already undergone multiple craniotomies and radiation. Repeat craniotomy or radiation could be considered, but each comes with inherent morbidities and the potential lack of long-term control. She was not interested in undergoing another craniotomy. In this case, MRgLITT was not only a minimally invasive approach, but it has also given her the longest recurrence-free interval she has experienced.

Case 2 had already undergone subtotal resection, and at recurrence chose to undergo MRgLITT as a minimally invasive alternative. It is unknown whether an open resection at that time, or radiation without MRgLITT, would have resulted in the same outcome. Given the eloquent location of the tumor recurrence, close attention was paid to limiting the amount of thermal energy given to the surrounding brain structures. As a result, a subtotal ablation was achieved with approximately $88 \%$ of the tumor being ablated. The tumor initially had a good response to MRgLITT, as evidenced by the decrease in tumor volume at the 6-month time point, but recurrence was noted at the 14-month follow-up evaluation. In her case, although MRgLITT was not curative, it provided a reduction in lesion size. Ultimately, surgical excision was the solution when she experienced recurrence after MRgLITT. Whether this patient could have been spared 2 procedures with initial resection instead of MRgLITT is not entirely clear, because on presentation previous resection had been subtotal. Ultimately, it may not be as relevant, because the patient at the time was unwilling to undergo another open resection. The experience of this patient underscores the point that subtotal ablation is analogous to a subtotal resection with respect to the likelihood of tumor recurrence.

The 2 pediatric cases represent fairly common situations in the treatment of ependymoma. The difficulty in decision making often comes from weighing the risks of a repeat craniotomy in a child who has already undergone multiple open procedures in a situation that will not likely result in long-term cure. In these situations, MRgLITT can be an extremely valuable tool. The first of these patients (Case 3) had exhausted all options, as is often the case, yet remained asymptomatic. The case was complex, with deep-seated lesions near critical structures. We were able to achieve palliative control for 10 months following his initial treatment. There are two obvious issues that cannot be resolved in this single case. The first is whether he would have survived just as long without an intervention or with another attempt at resection, given that one of the tumors was in the lateral ventricle and unlikely to become symptomatic until it grew much larger. The second issue is, after this patient's last MRgLITT procedure, he experienced irreversible endocrine disturbances after ablation. This neurological decline resulted in loss of independent functioning for the last 2 months of his life. It would be difficult to pinpoint the exact cause of neurological decline, because the same tumor was ablated safely twice before. The hypothesis is that the injury occurred either as a direct thermal injury to the pituitary gland, or an ischemic insult to the gland as a result of the destruction of feeding arteries. However, postinjury imaging did not reveal any ischemic insult to the pituitary gland. The third ventricular lesion had more than doubled its initial presentation volume by the time of the third ablation. With a much larger lesion to ablate, the risk for thermal injury was potentially greater. It is likely that we have much more to learn about change in tissue properties and environment after repeated ablations. It is possible that a lower power for thermal energy delivery is required in this location when a prior ablation has been performed. Also, newer software features such as multislice monitoring and contouring would increase the control of ablation in similar difficult locations. Despite the unfortunate outcome after the third MRgLITT procedure, the 7-month period of successive ablative procedures allowed the patient to spend a major portion of that time at home with his family. The repeated minimally invasive approach gave the patient a quality of life that may have been difficult to achieve with repeated resections.

The second pediatric patient (Case 4) represented a similar clinical situation but had a nodular recurrence at the margin of the operative cavity. For this lesion, radiation was not an option because the patient had already received maximal dosing. Therefore, the only options were either a sixth craniotomy or MRgLITT. In this case, we achieved ablation of the tumor without incurring morbidity from the procedure. Although there was no recurrence noted at the ablated site, with local control at 4 months, the patient developed CSF dissemination of his disease that could not be treated. At that point the patient was placed on hospice care and died 1 month later. For this case the same question arises: did the procedure extend the overall survival? The ethical question of whether to treat an asymptomatic recurrence in a child with this disease is not likely to be answered in the context of this study. The local control achieved in these cases may be dependent on the amount of ablated tumor. This leads us to the second purpose of this study, which was to examine the utility of the real-time TDE and its relation to tumor control.

\section{The TDE}

The TDE is an important component of the Visualase MRgLITT system and we hypothesized that it may function as an indicator for likelihood of recurrence. In animal models, McNichols et al. demonstrated that the TDE is an accurate measure of ablation size, a feature that is particularly relevant to ependymomas because maximal cytoreduction is critical. ${ }^{21}$ This advance provides a key update over prior techniques, as the operator does not need to ablate, then image, then ablate again, as was the case previously. The "intra-ablation" imaging becomes complicated, and an assessment of true ablation size is difficult until imaging is performed over the following days. The real-time TDE allows this assessment to occur during the ablation, rather than after. 
Although it appears intuitive that the TDE and the volume of tumor ablated should be directly proportional, we felt the need to provide proof of that correlation, as most treatments are conducted in a single plane without realtime visualization of the entire volume of the tumor. The next step was normalizing the TDE to the initial tumor size, which we call the PAA. Again, although seemingly intuitive, we show that the PAA correlates with the PVA in this series of patients/tumors. Through analysis of the PAA and PVA for each case, we found that the extent of intraoperative ablation may correlate with longer recurrence-free intervals. Based on the TDE, both Case 1 and Case 4 had PAA and PVA values exceeding 95\%. Case 1 achieved a virtual cure; however, the patient in Case 4 died from disseminated disease before sufficient follow-up. In contrast, Case 3 had values in the range of $40 \%-87 \%$, with only the PAA at the final ablation reaching $87 \%$. Case 2 had values approaching $88 \%$. Correspondingly, both patients had recurrences, with Case 2 having a longer time to recurrence. The resultant correlation $\mathrm{r}$ values reflected this association, with PAA and PVA predictive of recurrence likelihood. There is no question that the strength of the correlation is based on extremely small sample sizes, but the only 2 tumors that achieved reasonable local control had a PAA near or above $100 \%$. It appears that the time to recurrence was similar with an ablation range of $40 \%-60 \%$, so that there may be a threshold for ablation volume that is necessary to achieve longer control. This should serve as a foundation for future studies with larger sample sizes.

\section{Utilizing LITT and Study Limitations}

General considerations for MRgLITT also extend to treatment for ependymoma. Factors such as lesion size, geometry, and anatomical location, in addition to the tumor's perfusion characteristics, all likely play a role in the ability to achieve a complete ablation. The first patient had a relatively small lesion $\left(<1.0 \mathrm{~cm}^{3}\right)$, while the others had significantly larger lesions (ranging from 3.1 to 17.9 $\left.\mathrm{cm}^{3}\right)$. Although previous studies have reported successful MRgLITT for larger lesions, it has been our experience that the likelihood of a complete ablation is much more likely with smaller tumors. ${ }^{3,29}$ For larger lesions (> $3 \mathrm{~cm}^{3}$ ) and multiple lesions, the acute postablation edema is an important event that needs to be considered when deciding the size of ablation. This is similar to the tumor size limitations for stereotactic radiation therapy. The 3D shape of a lesion is a critical point and lesions not within the geometry of a first-order sphere or ellipsoid shape may not have ideal thermal energy distribution. ${ }^{3,29}$ Although multiple laser catheters may be useful for treating lesions with complex sizes and geometries, lesion location likely plays a role in response to MRgLITT. The second and third cases both involved lesions with CSF nearby, which likely acts as a heat sink preventing the spread of thermal energy. Thus, the differences in response among the cases are likely due to a combination of variables.

As with any small retrospective investigation, our study has the same limitations that one would expect from its design. This study represents the first time that the realtime TDE has been investigated in a clinical study, and more robust study designs will be necessary to determine its true utility. Further studies will be needed to identify specific PAA values, as identified by the TDE, at which a surgeon can stop the MRgLITT procedure. One patient experienced irreversible neurological deficits after the third procedure, which underscores the importance of patient selection, understanding of tumor anatomy, use of more monitoring planes, and tissue changes from prior ablations.

Ependymoma is often a disease of a younger age group, and our study included the age range of 13-59 years. Whether MRgLITT is safe for even younger patients remains to be shown. While MRgLITT may be used successfully in the short term, it has yet to be proven to provide long-term benefit. Nonetheless, our study demonstrates that MRgLITT may be used successfully in selected cases of ependymoma with nodular recurrence when other treatment options are no longer viable or when palliation is the goal. Larger studies will help better define the role of MRgLITT in this evolving treatment paradigm. Furthermore, the minimally invasive approach offers the advantages of repeatability and ability to reach deep areas in the brain safely.

\section{Conclusions}

This study demonstrates various clinical scenarios associated with recurrent ependymomas in which consideration of MRgLITT may be useful. It is possible to achieve longterm control for these tumors, but careful consideration must be given to the tumor's size and location. Subtotally ablated ependymomas will recur. The real-time TDE is a useful software advent that gives the surgeon confidence in the level of ablation and corresponding likelihood of recurrence. Further studies will be needed to define the future role of MRgLITT in the treatment of ependymomas.

\section{References}

1. Ascher PW, Justich E, Schröttner O: A new surgical but less invasive treatment of central brain tumours. Preliminary report. Acta Neurochir Suppl (Wien) 52:78-80, 1991

2. Bhuyan BK: Kinetics of cell kill by hyperthermia. Cancer Res 39:2277-2284, 1979

3. Bouffet E, Capra M, Bartels U: Salvage chemotherapy for metastatic and recurrent ependymoma of childhood. Childs Nerv Syst 25:1293-1301, 2009

4. Bouffet E, Perilongo G, Canete A, Massimino M: Intracranial ependymomas in children: a critical review of prognostic factors and a plea for cooperation. Med Pediatr Oncol 30:319-331, 1998

5. Bown SG: The future of lasers in cancer therapy. Br J Hosp Med 40:161, 1988

6. Carpentier A, McNichols RJ, Stafford RJ, Itzcovitz J, Guichard JP, Reizine D, et al: Real-time magnetic resonance-guided laser thermal therapy for focal metastatic brain tumors. Neurosurgery 63 (1 Suppl 1):ONS21-ONS29, 2008

7. Dohrmann GJ, Farwell JR, Flannery JT: Ependymomas and ependymoblastomas in children. J Neurosurg 45:273-283, 1976

8. Foreman NK, Love S, Thorne R: Intracranial ependymomas: analysis of prognostic factors in a population-based series. Pediatr Neurosurg 24:119-125, 1996

9. Hata N, Morrison PR, Kettenbach J, Black PM, Kikinis R, Jolesz FA: Computer-assisted intra-operative magnetic reso- 
nance imaging monitoring of interstitial laser therapy in the brain: a case report. J Biomed Opt 3:304-311, 1998

10. Heisterkamp J, van Hillegersberg R, Zondervan PE, IJzermans JN: Metabolic activity and DNA integrity in human hepatic metastases after interstitial laser coagulation (ILC). Lasers Surg Med 28:80-86, 2001

11. Iqbal MS, Lewis J: An overview of the management of adult ependymomas with emphasis on relapsed disease. Clin Oncol (R Coll Radiol) 25:726-733, 2013

12. Jethwa PR, Barrese JC, Gowda A, Shetty A, Danish SF: Magnetic resonance thermometry-guided laser-induced thermal therapy for intracranial neoplasms: initial experience. Neurosurgery 71:133-145, 2012

13. Jolesz FA, Bleier AR, Jakab P, Ruenzel PW, Huttl K, Jako GJ: MR imaging of laser-tissue interactions. Radiology 168:249-253, 1988

14. Kano H, Niranjan A, Kondziolka D, Flickinger JC, Lunsford LD: Outcome predictors for intracranial ependymoma radiosurgery. Neurosurgery 64:279-288, 2009

15. Kano H, Yang HC, Kondziolka D, Niranjan A, Arai Y, Flickinger JC, et al: Stereotactic radiosurgery for pediatric recurrent intracranial ependymomas. J Neurosurg Pediatr 6:417-423, 2010

16. Kawabata Y, Takahashi JA, Arakawa Y, Hashimoto N: Longterm outcome in patients harboring intracranial ependymoma. J Neurosurg 103:31-37, 2005

17. Kettenbach J, Silverman SG, Hata N, Kuroda K, Saiviroonporn P, Zientara GP, et al: Monitoring and visualization techniques for MR-guided laser ablations in an open MR system. J Magn Reson Imaging 8:933-943, 1998

18. Lindner U, Trachtenberg J, Lawrentschuk N: Focal therapy in prostate cancer: modalities, findings and future considerations. Nat Rev Urol 7:562-571, 2010

19. Lo SS, Chang EL, Sloan AE: Role of stereotactic radiosurgery and fractionated stereotactic radiotherapy in the management of intracranial ependymoma. Expert Rev Neurother 6:501-507, 2006

20. McGuire CS, Sainani KL, Fisher PG: Incidence patterns for ependymoma: a surveillance, epidemiology, and end results study. J Neurosurg 110:725-729, 2009

21. McNichols RJ, Gowda A, Kangasniemi M, Bankson JA, Price RE, Hazle JD: MR thermometry-based feedback control of laser interstitial thermal therapy at $980 \mathrm{~nm}$. Lasers Surg Med 34:48-55, 2004

22. Nazar GB, Hoffman HJ, Becker LE, Jenkin D, Humphreys RP, Hendrick EB: Infratentorial ependymomas in childhood: prognostic factors and treatment. J Neurosurg 72:408-417, 1990

23. Nejat F, El Khashab M, Rutka JT: Initial management of childhood brain tumors: neurosurgical considerations. J Child Neurol 23:1136-1148, 2008

24. Patel NV, Jethwa PR, Barrese JC, Hargreaves EL, Danish SF: Volumetric trends associated with MRI-guided laser-induced thermal therapy (LITT) for intracranial tumors. Lasers Surg Med 45:362-369, 2013

25. Pollack IF, Gerszten PC, Martinez AJ, Lo KH, Shultz B, Albright AL, et al: Intracranial ependymomas of childhood: long-term outcome and prognostic factors. Neurosurgery 37:655-667, 1995

26. Pushek T, Farahani K, Saxton RE, Soudant J, Lufkin R, Paiva M, et al: Dynamic MRI-guided interstitial laser therapy: a new technique for minimally invasive surgery. Laryngoscope 105:1245-1252, 1995
27. Schild SE, Nisi K, Scheithauer BW, Wong WW, Lyons MK, Schomberg PJ, et al: The results of radiotherapy for ependymomas: the Mayo Clinic experience. Int J Radiat Oncol Biol Phys 42:953-958, 1998

28. Schulze PC, Adams V, Busert C, Bettag M, Kahn T, Schober $\mathrm{R}$ : Effects of laser-induced thermotherapy (LITT) on proliferation and apoptosis of glioma cells in rat brain transplantation tumors. Lasers Surg Med 30:227-232, 2002

29. Schwarzmaier HJ, Eickmeyer F, von Tempelhoff W, Fiedler VU, Niehoff H, Ulrich SD, et al: MR-guided laser-induced interstitial thermotherapy of recurrent glioblastoma multiforme: preliminary results in 16 patients. Eur J Radiol 59:208-215, 2006

30. Sercarz JA, Bublik M, Joo J, Paiva PB, Areco KN, Brandalise $\mathrm{MH}$, et al: Outcomes of laser thermal therapy for recurrent head and neck cancer. Otolaryngol Head Neck Surg 142:344-350, 2010

31. Shaw EG, Evans RG, Scheithauer BW, Ilstrup DM, Earle JD: Postoperative radiotherapy of intracranial ependymoma in pediatric and adult patients. Int J Radiat Oncol Biol Phys 13:1457-1462, 1987

32. Stauder MC, Ni Laack N, Ahmed KA, Link MJ, Schomberg PJ, Pollock BE: Stereotactic radiosurgery for patients with recurrent intracranial ependymomas. J Neurooncol 108:507-512, 2012

33. Sutton LN, Goldwein JW, Schwartz D: Ependymomas, in Albright AL, Adelson PD, Pollack IF: (eds): Principles and Practice of Pediatric Neurosurgery. New York: Thieme, 1999, pp 609-628

34. Tatter SB, Wilson CB, Harsh GR IV: Neuroepithelial tumors of the adult brain, in Youmans JR (ed): Neurological Surgery, ed 4. Philadelphia: Saunders, 1995, Vol 4, pp 26122684

35. Teo C, Nakaji P, Symons P, Tobias V, Cohn R, Smee R: Ependymoma. Childs Nerv Syst 19:270-285, 2003

36. van Esser S, Stapper G, van Diest PJ, van den Bosch MA, Klaessens JH, Mali WP, et al: Ultrasound-guided laserinduced thermal therapy for small palpable invasive breast carcinomas: a feasibility study. Ann Surg Oncol 16:2259_ 2263, 2009

37. Vogl TJ, Naguib NN, Eichler K, Lehnert T, Ackermann H, Mack MG: Volumetric evaluation of liver metastases after thermal ablation: long-term results following MR-guided laser-induced thermotherapy. Radiology 249:865-871, 2008

\section{Author Contributions}

Conception and design: Patel, Jethwa, Danish. Acquisition of data: Patel, Jethwa, Danish. Analysis and interpretation of data: Patel, Jethwa, Danish. Drafting the article: all authors. Critically revising the article: Patel, Danish. Reviewed submitted version of manuscript: Patel, Danish. Approved the final version of the manuscript on behalf of all authors: Patel. Statistical analysis: Patel, Jethwa. Administrative/technical/material support: all authors. Study supervision: Patel, Jethwa, Danish.

\section{Correspondence}

Nitesh V. Patel, Division of Neurosurgery, Rutgers-Robert Wood Johnson Medical School, 125 Paterson St., CAB Ste. 2100, New Brunswick, NJ 08901. email: patel236@rwjms.rutgers.edu. 